02.2;09.1

\title{
Возбуждение молекул валина электронным ударом в газовой фазе
}

\author{
() О.Б. Шпеник, А.И. Булгакова , А.Н. Завилопуло, Н.М. Эрдевди, Ю.А. Бандурин \\ Институт электронной фризики НАН Украины, Ужгород, Украина \\ `E-mail: alla.bulgakova.uzh@gmail.com
}

Поступило в Редакцию 6 апреля 2021 г.

В окончательной редакции 6 апреля 2021 г.

Принято к публикации 20 апреля 2021 г.

Описаны методика измерений и результаты, полученные методом оптической спектроскопии при возбуждении молекул валина в газовой фазе медленными электронами. Приведены оптические спектры излучения исследуемых молекул в области $260-440 \mathrm{~nm}$ при возбуждении электронами в интервале энергий $10-70 \mathrm{eV}$. Для наиболее интенсивных спектральных линий (полос) излучения измерены оптические функции возбуждения электронным ударом в диапазоне энергий 3-90 eV.

Ключевые слова: электрон, валин, возбуждение, фрагментация.

DOI: 10.21883/PJTF.2021.14.51184.18810

Ионизирующее излучение, взаимодействуя с живым организмом, может вызывать различные изменения в генотипе вследствие воздействия на генетические макромолекулы ДНК и РНК. Это излучение, проникая в организм, генерирует потоки низкоэнергетических вторичных электронов, энергия которых может составлять от 0.1 до десятков $\mathrm{eV}$ [1]. Вторичные электроны инициируют процессы возбуждения [2,3] и ионизации [4], которые приводят к деструктивным изменениям в ДНК и РНК [5]. Оценка последствий физических процессов возбуждения и ионизации, вызванных в биоструктуpax электронным ударом, требует получения информации о наиболее вероятных каналах фрагментации биомолекул.

Возбуждение молекул медленными электронами сопровождается переходом одного или нескольких электронов исходной молекулы на вышестоящие электронные состояния. Последующее девозбуждение приводит к появлению в спектрах излучения как молекулярных полос самой молекулы, так и ее фрагментов, а также спектральных линий отдельных атомов (например, водорода), охватывающих широкий диапазон длин волн от инфракрасного до видимого и ультрафиолетового [6].

Естественно, что наиболее эффективным методом в таких исследованиях является оптический, а применение моноэнергетических пучков электронов при возбуждении сложных молекул в газовой фазе позволяет получить информацию о положении и структуре энергетических уровней, а также оценить относительную вероятность их возбуждения. Отметим, что исследование молекул в газовой фазе позволяет исключить сольватационные эффекты, которые могут влиять на конформационную стабильность молекул и перераспределение колебательных возбуждений в реальных биоорганических системах.
По сложившимся представлениям [7] именно с медленными электронами связывают основную часть деструктивных изменений на молекулярном уровне биоструктур, причем главной мишенью являются генетические макромолекулы [8]. Актуальность исследования этих процессов различными методами в особенности для случая биомолекул приобрела в последнее время особое значение в связи с пандемией COVID-19 $[9,10]$.

В настоящей работе исследованы спектры излучения молекул валина в газовой фазе при возбуждении электронами в интервале энергий $10-70 \mathrm{eV}$, а также оптические функции возбуждения (ОФВ) отдельных полос (линий) излучения в интервале энергий 5-90eV и проведена идентификация механизмов их возбуждения.

Валин относится к группе простых алифатических неполярных $\alpha$-аминокислот, которые имеют наибольшую алкильную боковую цепь $\mathrm{C}_{\alpha}$ и существуют в виде различных конформеров. Известны два вида изомерии аминокислот: структурная, которая связана с особенностями строения углеродного скелета и взаимным расположением функциональных групп, и оптическая изомерия (пространственная). Поскольку $\alpha$-аминокислоты содержат асимметрический атом углерода $\left(\mathrm{C}_{\alpha}\right.$-атом $)$, они могут существовать в виде оптических изомеров (зеркальных антиподов), которые играют важную роль в процессах биосинтеза белка.

На рис. 1 представлены структурные схемы молекулы валина $\mathrm{C}_{5} \mathrm{H}_{11} \mathrm{NO}_{2}$. Следует заметить, что алифатические аминокислоты можно рассматривать как модельные системы в процессах взаимодействия ионизирующего излучения с более крупными биомолекулярными комплексами (белки, пептиды). Под воздействием первичного интенсивного ионизирующего излучения возникают вторичные частицы, такие как радикалы или электроны с энергией ниже $20 \mathrm{eV}$, играющие важную 


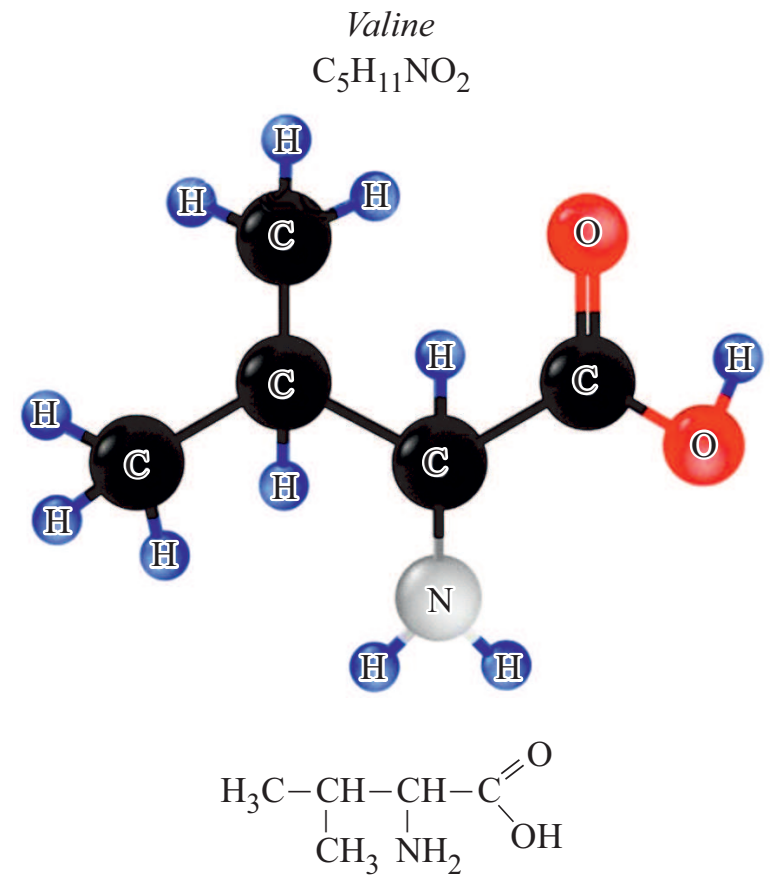

Pис. 1. Структурные формулы молекулы валина.

роль в генерации дефектов в биологической системе. Величины энергии вторичных электронов достаточно, чтобы вызвать непосредственно в среде процессы возбуждения, диссоциации, прилипания и/или электронную ионизацию, что инициирует дополнительные повреждения структуры.

Как следует из рис. 1, отличительной особенностью молекулы валина является наличие атома углерода, который связан с четырьмя заместителями: один из них - атом водорода, второй - карбоксильная группа $-\mathrm{COOH}$, третий - аминогруппа $-\mathrm{NH}_{2}$, способная присоединять ион водорода, четвертый - алкильная боковая цепь $\mathrm{C}_{\alpha}$-атомов, состав которой определяет основные свойства данной аминокислоты. Энантиомеры валина имеют одинаковые энергетические характеристики (потенциалы возбуждения, ионизации, теплоты образования, энергии активации) и отличаются только векторными характеристиками [7].

Карбоксильная группа может вращаться, а атом водорода - ориентироваться как в направлении азота, так и в обратном направлении. Кроме того, конформационная изменчивость молекул способствует переориентировке гибкой карбоксильной $(-\mathrm{COOH})$ и аминной $\left(-\mathrm{NH}_{2}\right)$ групп (рис. 1) с образованием различных внутримолекулярных водородных связей, которые, например, соединяют неразделенную пару атомов азота с водородом гидроксильной группы или же устанавливают связь между атомом водорода аминной группы и атомом кислорода карбонильной $(\mathrm{NH} \ldots \mathrm{O}=\mathrm{C})$ и гидроксильной $(\mathrm{NH} \ldots \mathrm{OH})$ групп.
Эксперимент, подробно описанный в [11], проводился с применением паронаполненной ячейки, в которой с использованием ампулы с независимым нагревом создавалась концентрация молекул валина в области взаимодействия с электронами порядка $6 \cdot 10^{11} \mathrm{~cm}^{-3}$. Пучок электронов диаметром $2 \mathrm{~mm} \mathrm{c}$ силой тока $10-100 \mu \mathrm{A}$ в интервале энергий $0-90 \mathrm{eV}$ детектировался цилиндром Фарадея. Моноэнергетичность электронного пучка (полная ширина на полувысоте) была не хуже $\Delta E_{1 / 2}=0.5 \mathrm{eV}$ и определялась автоматически перед каждым измерением. Энергия электронов при измерениях оптических функций возбуждения в заданном интервале энергий сканировалась автоматизированной системой измерений с шагом $100-400 \mathrm{meV}$.

Излучение из ячейки столкновений через кварцевое окно в боковой стенке вакуумной камеры с помощью линзы фокусировалось на входную щель монохроматора МДР-2 и детектировалось фотоэлектронным умножителем (ФЭУ). Система автоматического контроля параметров и регистрации полезного сигнала позволяла работать в режиме счета фотонов и направлять электронные импульсы с ФЭУ для обработки и записи на персональный компьютер. Оптические спектры излучения в диапазоне длин волн $\lambda=260-440 \mathrm{~nm}$ измерялись с шагом $0.547 \mathrm{~nm}$, время экспозиции накопления сигнала выбиралось в интервале $1-5 \mathrm{~s}$ в каждой точке, а входная щель монохроматора шириной $1 \mathrm{~mm}$ обеспечивала спектральное разрешение $\Delta \lambda=2 \mathrm{~nm}$.

Для корректной интерпретации результатов измерений калибровка энергетической шкалы пучка возбуждающих электронов осуществлялась двумя независимыми методами: по смещению вольт-амперной характеристики электронного тока на коллектор и по порогу возбуждения резонансной линии $\mathrm{Hg} \mathrm{c} \lambda=253.7 \mathrm{~nm}$ (величина энергии $E_{t h r}=4.885 \mathrm{eV}$ ) и по положению резкого максимума $E=14.224 \mathrm{eV}$ на ОФВ спектральной полосы $\mathrm{N}_{2}$ с $\lambda=337.1 \mathrm{~nm}\left(C^{3} \Pi_{u} \rightarrow B^{3} \Pi_{g}\right)$. Калибровка шкалы барабана спектрального прибора проводилась по спектральным линиям атома ртути с $\lambda=253.7,334.1$ и $365.0 \mathrm{~nm}$. Таким образом, установленная нами неопределенность шкалы энергий электронов при измерении ОФВ была не хуже $\pm 0.1 \mathrm{eV}$, а шкалы спектрального прибора - не хуже $\pm 0.25 \mathrm{~nm}$.

Процедура измерений включала в себя два этапа: на первом записывались спектры излучения молекул валина при фиксированных энергиях электронов 30, 50 и $70 \mathrm{eV}$ в диапазоне длин волн 260-440 nm (рис. 2), а на втором измерялись ОФВ наиболее интенсивных эмиссий как молекулярных полос излучения, так и атомарных линий (рис. 3).

При энергии возбуждающих электронов $E_{\text {exc }}=30 \mathrm{eV}$ в оптическом спектре присутствуют две яркие полосы излучения вблизи 280 и $307 \mathrm{~nm}$ и несколько слабых эмиссий, которые расположены в более длинноволновой 

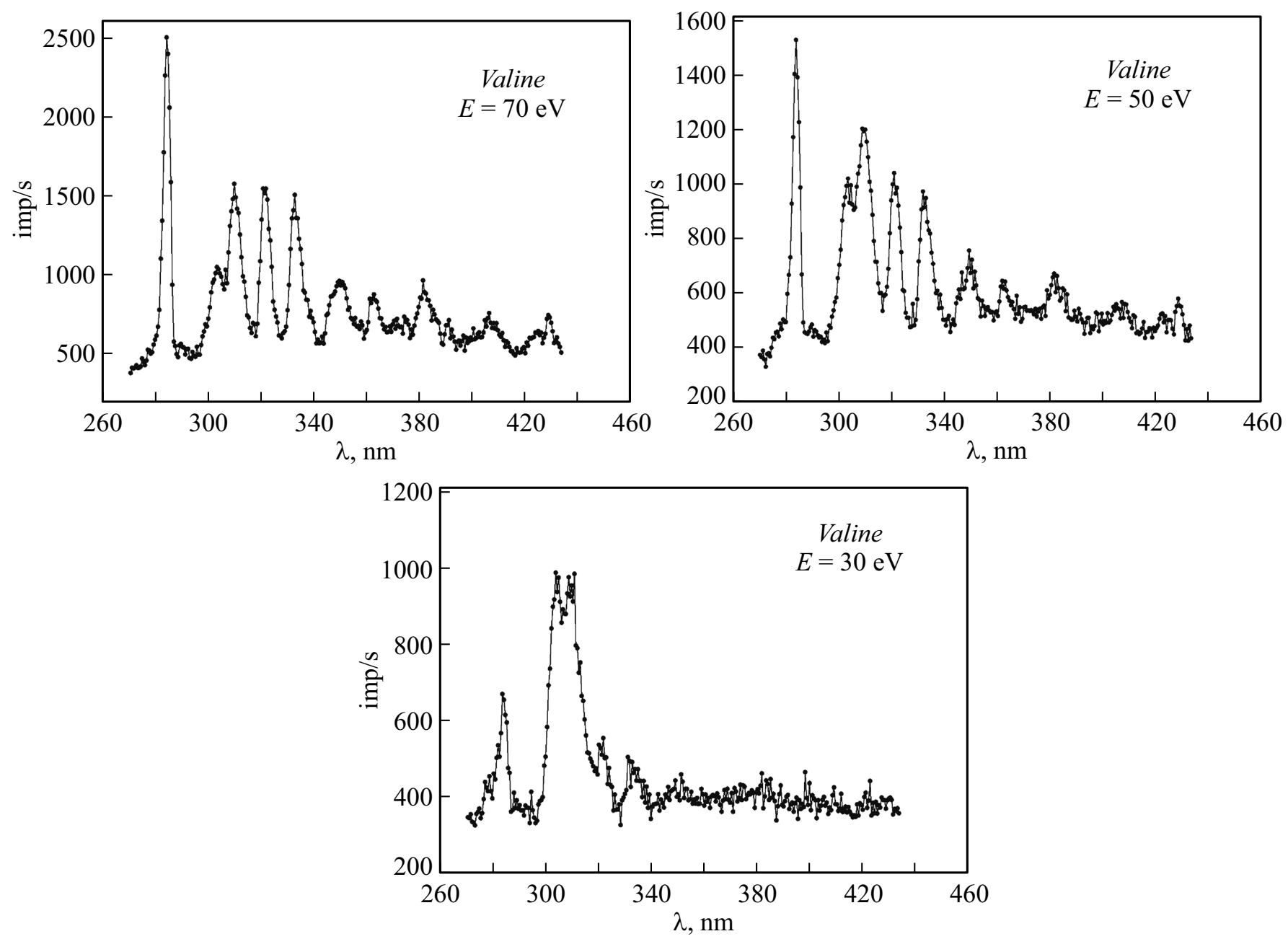

Рис. 2. Спектры излучения при энергиях возбуждающих электронов 30,50 и $70 \mathrm{eV}$.

области спектра. Далее с ростом энергии электронов наблюдается общее увеличение эмиссии фотонов, а наиболее резко возрастает интенсивность излучения коротковолновой полосы вблизи $280 \mathrm{~nm}$, она становится доминирующей в спектре. В длинноволновой области спектра более „выразительными“ становятся полосы излучения при $321,332,350,362.5,381,406.5$ и $429 \mathrm{~nm}$ (рис. 2).

Сложная структура молекулы валина (рис. 1) и имеющиеся в литературе данные [12] позволяют однозначно идентифицировать лишь часть из наблюдаемых в спектре молекулярных полос. В спектре при $E_{\text {exc }}=30 \mathrm{eV}$ основной вклад в излучение довольно широкой полосы с $\lambda=307 \mathrm{~nm}$ вносит возбужденный радикал ОН (переход $A^{2} \sum^{+}-X^{2} \Pi i$, полоса $\left.0-0\right)$, излучение которого расщепляется на 306.5, 306.8 и $309.2 \mathrm{~nm}$. Девозбуждение этой системы с колебательными уровнями 1-1 проявляется на длинах волн $\lambda=312.5+314.5 \mathrm{~nm}$, а с колебательными уровнями 2-2 эффективно проявляется на длинах волн $\lambda=318.5,321,323 \mathrm{~nm}$, что и наблюдается в спектре (рис. 2). Кроме того, де- тальное изучение спектра излучения радикала ОН [13] позволяет идентифицировать также переходы данной системы с колебательными уровнями 1-0, которые приводят к появлению полос с $\lambda=281.2,283 \mathrm{~nm}$ и наиболее интенсивной полосы с $\lambda=284 \mathrm{~nm}$. Можно также утверждать, что в измеренном спектре наблюдается и незначительное проявление полосы системы $B^{2} \sum^{+}-A^{2} \sum^{+}$с $\lambda=278.04 \mathrm{~nm}$ в виде коротковолнового „уступа“. Это неудивительно, поскольку минимальная энергия возбуждения уровня $A^{2} \sum^{+}$ составляет $4.052 \mathrm{eV}$, а для уровня $B^{2} \sum^{+}$она равна $8.65 \mathrm{eV}$.

Вместе с тем обращает на себя внимание тот факт, что соотношение интенсивностей полос излучения молекулы ОН с колебательными уровнями 1-0 (283 nm) и 0-0 (307-309 nm) при других способах возбуждения [13] составляет $\sim 0.37$. В нашем случае при энергии электронов $30 \mathrm{eV}$ это соотношение равно 0.67 , при $50 \mathrm{eV}-1.28$, при $70 \mathrm{eV}$ - 1.58. Увеличение энергии налетающих электронов не должно приводить к существенному изменению заселенностей возбужденных 

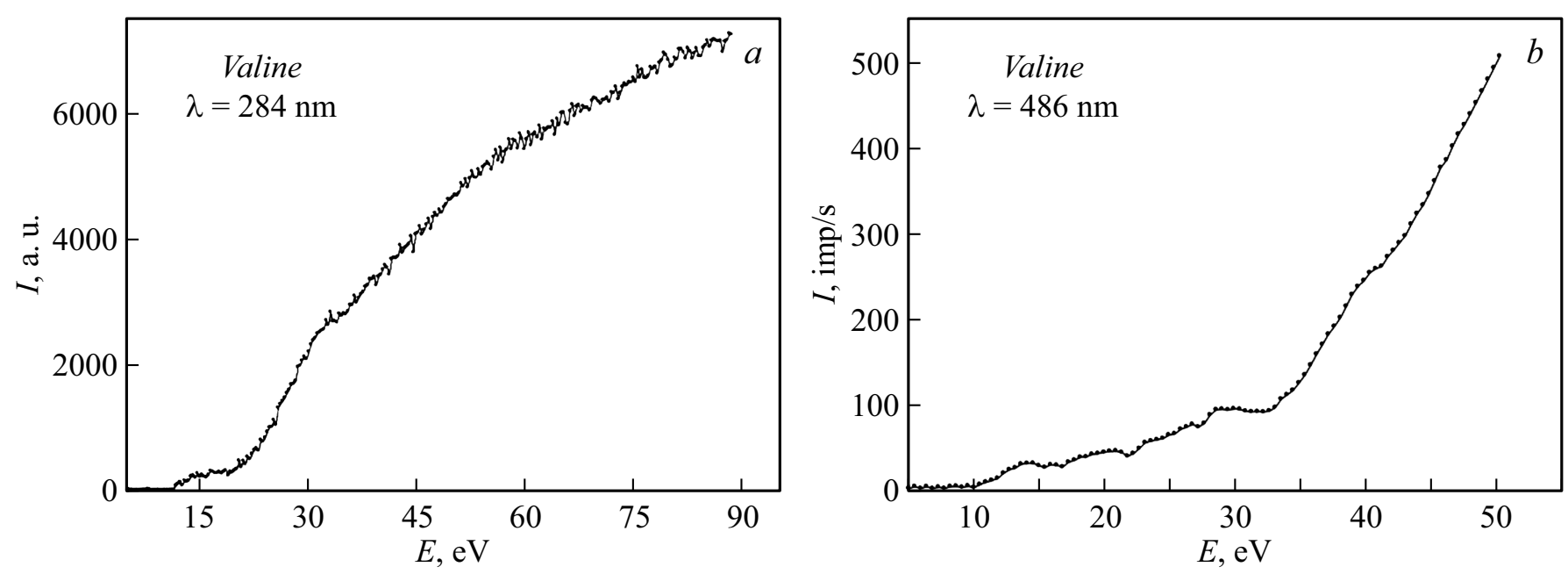

Рис. 3. Оптические функции возбуждения молекулы валина электронным ударом. $a-$ спектральная полоса с $\lambda=284 \mathrm{~nm}, b-$ спектральная линия атома водорода $\mathrm{H}_{\beta} \mathrm{c} \lambda=486 \mathrm{~nm}$.

состояний молекулы ОН, как и при других способах возбуждения [13]. Это означает, что в излучение на длине волны $\lambda=283 \mathrm{~nm}$, вероятно, вносит вклад полоса третьей положительной группы молекулы СО, переход $b^{3} \sum^{+}-a^{3} \Pi$ (уровень $0-0, \lambda=283 \mathrm{~nm}$ ). Обе возбужденные молекулы $\mathrm{OH}^{*}$ и $\mathrm{CO}^{*}$ несомненно являются фрагментами, образованными в результате электронного удара по молекуле валина. Увеличение энергии бомбардирующих электронов с 30 до $70 \mathrm{eV}$ приводит к перераспределению доли каждого из этих фрагментов, что проявляется в неодинаковом изменении интенсивности излучения. Для проверки этого предположения мы провели измерения оптических функций возбуждения как для этих молекулярных полос для длин волн $\lambda=284,311,335 \mathrm{~nm}$, так и для спектральной линии атома водорода $\mathrm{H}_{\beta}(\lambda=486 \mathrm{~nm})$. На рис. 3 в качестве примера показаны ОФВ, измеренные для молекулярной полосы с $\lambda=284$, а также для спектральной линии атома водорода $\mathrm{H}_{\beta}$ с $\lambda=486 \mathrm{~nm}$. Определенные нами энергетические пороги возбуждения всех видов эмиссий составляют $10-11 \mathrm{eV}$. Очевидно, что в этом интервале энергий находится энергетический порог фрагментации молекулы валина. Увеличение квантового выхода с ростом энергии бомбардирующих электронов индивидуально для каждой эмиссии (полосы или линии), что свидетельствует об информативности подобных исследований процесса фрагментации.

В заключение отметим, что исследования аминокислот с помощью электронного удара в газовой фазе дают обширную информацию об их уникальных свойствах, позволяют определить пороги и величину степени фрагментации в процессе взаимодействия с электронами, оценить параметры межмолекулярных связей.

\section{Конфликт интересов}

Авторы заявляют, что у них нет конфликта интересов.

\section{Список литературы}

[1] L. Sanche, Rad. Phys. Chem., 128, 36 (2016). https://doi.org/10.1016/j.radphyschem.2016.05.008

[2] Н.М. Эрдевди, А.И. Булгакова, О.Б. Шпеник, А.Н. Завилопуло, Письма в ЖТФ, 46 (16), 35 (2020). DOI: 10.21883/PJTF.2020.16.49852.18287 [Пер. версия: 10.1134/S1063785020080209].

[3] А.Н. Завилопуло, А.И. Булгакова, Письма в ЖТФ, 45 (24), 36 (2019). DOI: 10.21883/PJTF.2019.24.48801.17968 [Пер. версия: 10.1134/S1063785019120290].

[4] A.N. Zavilopulo, O.B. Shpenik, A.N. Mylymko, V.Yu. Shpenik, Int. J. Mass Spectr. 441, 1 (2019). https://doi.org/10.1016/j.ijms.2019.03.008

[5] I. Fabrikant, S. Eden, N.J. Mason., J. Fedor, Adv. Atom. Mol. Opt. Phys., 66, 545 (2017). https://doi.org/10.1016/bs.aamop.2017.02.002

[6] Y. Hu, E.R. Bernstein, Chem. Phys., 128 (16), 164311 (2008). https://doi.org/10.1063/1.2902980

[7] О.В. Смирнов, А.А. Басалаев, В.М. Бойцов, С.Ю. Вязьмин, А.Л. Орбели, М.В. Дубина, ЖТФ, 84 (11), 121 (2014). [Пер. версия: 10.1134/S1063784214110231].

[8] C. Sonntag, The chemical basis for radiation biology (Taylor \& Francis Press, London, 1987).

[9] В.В. Никифоров, Т.Г. Суранова, Т.Я. Чернобровкина, Я.Д. Янковская, С.В. Бурова, Архив внутренней медицины, 10 (2), 87 (2020).

DOI: $10.20514 / 2226-6704-2020-10-2-87-93$

[10] Б.К. Романов, Безопасность и риск фармакотерапии, 8 (1), 3 (2020). https://doi.org/10.30895/2312-7821-2020-8-1-3-8 
[11] Н.М. Эрдевди, В.В. Звенигородский, О.Б. Шпеник, Л.Г. Романова, Оптика и спектроскопия, 114 (1), 51 (2013). DOI: $10.7868 / \mathrm{S} 003040341301008 \mathrm{X}$

[Пер. версия: 10.1134/S0030400X13010086].

[12] К.-П. Хьюбер, Г. Герцберг, Константы двухатомных молекул(Мир, М., 1984), ч. 1, 2.

[13] C.G. Pariger, C.M. Helstern, B.S. Jordan, D.M. Surmick, R. Splinter, Molecules, 25 (4), 988 (2020). http://doi:10.3390/molecules25040988 